Paper ID \#18457

\title{
PIPELINES: Fostering University-Community College Partnerships and STEM Professional Success for Underrepresented Populations
}

\author{
Dr. Maria Teresa Napoli, University of California, Santa Barbara
}

Dr. Maria Teresa Napoli received a Ph.D. degree in Mechanical Engineering from the University of California at Santa Barbara, in 2004. In 1999, she also earned a Ph.D. degree in electrical engineering from the University of Padova in Italy. Currently, she holds positions as VP of MEMS Development at Laxmi Therapeutic Devices, and as Community College Programs Manager at the University of California at Santa Barbara. Prior to this appointment, she worked for several years as a microsensors system expert at SensorDynamics AG. Her research interests include nanofluidic technologies for medical applications, modeling and control of large arrays of MEMS, and educational strategies and programs to increase STEM diversity.

Dr. Elizabeth Sciaky, University of California, Santa Barbara, Center for Science and Engineering Partnerships

Program Evaluator at the Center for Science and Engineering Partnerships at UC Santa Barbara.

Prof. Diana Jaleh Arya, University of California, Santa Barbara

Diana Arya is an assistant professor in the Department of Education at the University of California, Santa Barbara. Arya's research interests focus on science and engineering literacy practices within K-12 science classroom and professional communities.

\section{Ms. Noreen Balos, University of California, Santa Barbara}

Noreen Balos is a doctoral student in the Learning, Culture \& Technology program at the University of California, Santa Barbara (UCSB). Prior to UCSB, she served as Student Affairs Officer for UCLA's Biomedical Research minor program advising undergraduate researchers in their pursuit of MD or MDPhD. At ASU's School for Engineering of Matter, Transport, \& Energy (SEMTE), she was a Project Manager, overseeing with CO-PIs, an NSF Innovation through Institutional Integration (I^3) grant collaborating with academic departments such as mathematics, physics, engineering, and education. One of the foci of this grant was to train K-8 certified teachers in modeling pedagogy and to support them in receiving a Master's in STEM education. Her specific work with student STEM programs includes: connecting and funding K-12 students in university summer programs, coordinating STEM professional development programs for teachers, and leading departmental involvement in campus-based, state-wide Science and Technology festival for students, families, and the community. Prior to her work in higher education, Noreen was an Instructor Meteorologist \& Wing Weather Officer for the USAF, specifically training personnel in synoptic forecasting and providing operational weather data for different missions at home station and while on deployments to Europe and the Middle East. With her background in science and professional experience in higher education, her research interests include: STEM Education, Culture in STEM, and Access \& Equity in STEM. 


\title{
PIPELINES: Fostering University-Community College Partnerships and STEM Professional Success for Underrepresented Populations
}

\begin{abstract}
A survey of literature reveals a major problem in retaining engineering college students due to the perceived lack of connections with, and real access to STEM-related careers. This effect appears more pronounced for underrepresented groups, often first generation college students, including veterans. PIPELINES, a collaboration between a Navy Base in Southern California, a tier-1 research university and a network of community colleges, is an early workforce learning experience that fosters students' career preparedness while providing the ground to investigate the complexities of developing STEM readiness of underrepresented populations through an interactive ethnographic approach.

In this work, we highlight strategies we found effective in developing and implementing this multi-tiered, interdisciplinary effort, in which each actor (educators, researchers, and Navy scientists and engineers) brings complementary knowledge and skills that are key to PIPELINES programmatic and recruitment success. Additionally, lessons learned from our first iteration of the PIPELINES program are illustrated using findings from program evaluation (a separate and independent component from the ethnographic research study).
\end{abstract}

\section{Introduction}

A number of reports have highlighted the need to increase the number of students prepared for careers in STEM fields, indicated by all as an essential factor to maintain U.S. intellectual and economic competitiveness [1]-[4]. Against this backdrop, the Navy, who according to a recent study commissioned by the Office of Naval Research (ONR), is the most dependent on bachelor's-level employees of all the U.S. military branches [5], expects $50 \%$ of their civilian STEM workforce to be eligible for retirement by 2020 [5]. This imminent nationwide shortage of STEM Naval workers is already realized at the local Naval Base at Port Hueneme (Oxnard, CA), which currently has over 100 open STEM positions.

In spite of this surge in demand, recent trends show that the number of students graduating with STEM degrees is not growing accordingly [1], [6], [7]. It is undisputed that engaging the participation of underrepresented populations will prove crucial to ensure a highquality future science and engineering workforce [8]. Likewise, engagement of veteran students will also play a critical role. Military veterans are returning to school in very large numbers to enhance their career prospects; in 2012 (more recent data available), more than 900,000 veterans had used the education benefits offered through the Post-9/11 G.I. Bill [9], and more are expected to do so in the next several years [10]. Importantly, studies show that veterans gain advanced technical skills during their years of service, as well as workplace-relevant skills such as leadership and time management [11]-[14].

Unfortunately, it is our observation, supported by conversations with veteran coordinators at Community Colleges (CCs) and veteran advocates in our area that these often first-generation students frequently tend to opt for non-STEM degrees and jobs. The reasons behind this choice are multiple, and include a surprising lack of awareness by veterans about how their training and 
technical capacity might translate into STEM career opportunities [15], "rusty" mathematics skills, family commitments, concerns about time to degree completion [15], and lack of awareness about STEM career opportunities [16].

The Problem-based Initiatives for Powerful Engagement and Learning In Naval Engineering and Science (PIPELINES) aims at bridging this disconnect by offering a workforce learning experience that supports students' awareness and development of career-critical skills, while also highlighting potential civilian career pathways in the Navy. In addition, the PIPELINES program is a unique opportunity for us to investigate the complexities of developing STEM readiness of underrepresented populations through an interactive ethnographic approach. In the program, teams of undergraduate students ( 2 from community colleges, 1 from a 4-year university) are challenged to solve real-world Navy engineering design problems over a period of eight weeks during the summer. Each team is assigned a UCSB graduate student, from a relevant STEM field, and a Navy engineer that serve as mentors. From Monday through Thursday, student teams work at the Base in Port Hueneme on their separate projects. On Fridays, students come to UCSB to attend career exploration and professional development seminars, as well as a course in engineering innovation, designed to stimulate creative thinking and problem-solving. The program culminates with a Design Challenge Award, where teams pitch their project to a jury of faculty and Base engineers.

Key components that differentiate PIPELINES from similar initiatives and that we believe are key to its impact are: 1) fostering STEM diversity through an interdisciplinary partnership; 2) workforce learning through STEM design experiences; 3) leveraging teamwork for early engagement in engineering design. We discuss each one in the following sections.

\section{Fostering STEM Diversity through an Interdisciplinary Partnership}

Funded by ONR, PIPELINES is a collaborative effort between the Center for Science and Engineering Partnerships (CSEP) at the University of California Santa Barbara (UCSB), the Gevirtz Graduate School of Education at UCSB (GGSE), and the Naval Facilities Engineering Command, Navy Engineering and Expeditionary Warfare Center (NAVFAC EXWC) at Port Hueneme, California.

This interdisciplinary partnership that includes outreach professionals, education researchers and naval engineers is a crucial aspect that differentiates PIPELINES. Bringing such a diverse team together required the dedication and commitment of all partners to find a common language to communicate ideas, the belief that each partner brought unique value to the project, and the flexibility to adapt known work habits to a shared vision.

For instance, drawing interns from CCs was a new proposition for EXWC, who typically hires interns from 4-year universities. Having promoted programs targeting CCs students for over 20 years, CSEP outreach professionals championed the idea. California CCs serve a disproportionate number of underrepresented minority students: in 2010 (the most recent data available), CCs enrolled 1.75 million undergraduate students, of whom $45 \%$ were underrepresented minorities [14]. Similarly, in 2010-11 (the most recent data available) more than 44,000 veterans used education benefits at a California CC, an increase of about $70 \%$ compared to 2008-09 [16, 19]. This data suggests that CCs represent a significant opportunity to expand the pool of new recruits into STEM programs [17], [18]. Unfortunately, this potential pool of talent is often overlooked, due to a misguided perception of its students as being less 
capable [17]. This is a case where EXWC leadership and engineers trusted CSEP professionals with the decision.

End of the program evaluation findings show that Navy mentors made frequent mention of how impressed they were with the maturity and enthusiasm of their interns.

"In retrospect, having a group like that, if you get it, it's just a joy."

EXWC mentors were pleasantly surprised to find their teams composed of older, more mature students with a diverse array of personal, professional and academic experiences. In most cases, the interns exceeded the mentors' expectations, proving to be quick learners and motivated employees. Several suspected that the older age and greater life experience of the interns was a factor in their preparedness for the internship. In particular, mentors were impressed by their veteran interns, who tended to be leaders and/or practical thinkers in their respective groups. "Usually interns don't have any idea what our mission is, whereas if you have a veteran from Iraq and you're talking about the climate in Djibouti... one of my interns actually lived in the thing that we're building. That kind of real world experience is really valuable."

As another example, PIPELINES's team includes ethnographers, who seek to derive lessons that can inform a range of Navy and civilian practitioners and program developers to increase the number of underrepresented STEM professionals. In contrast to traditional program evaluation, the ethnographic methodology is not a fixed intervention and is based, instead, on an 'abductive logic' that requires the education research team to develop an emic (i.e., insider) perspective [19]. In practice, this means that the ethnographer needs frequent contact with all participants to gain insights into students' perceptions of science and engineering as a way of thinking and being. This interaction yields new lines of inquiry, shaped by observed stakeholder experiences, perspectives, and attitudes, systematically documented via recorded exchanges, discussions, individual and collaborative work products, and field notes. This approach represented a significant paradigm shift for program organizers and participants (both students and Navy mentors). However, at the end of the first year, we are discovering interesting connections between students' uptake of engineering concepts and their forming of an engineering identity, and the content and evolution of their journal writing (details will be discussed in a separate publication).

In short, PIPELINES is an example of how different entities can work together towards achieving their respective goals, all of which are focused on increasing a currently dwindling engineering workforce in the U.S.

\section{Workforce Learning through STEM Design Experiences}

PIPELINES Navy design experience emphasizes collaborative, problem-based learning, with a focus on Navy STEM problems. Such activities support and encourage students to develop skills, such as critical thinking and problem solving that are essential for academic and workplace success.

A survey of the literature reveals that engineering students leave their major mainly because the first year of an engineering program tend to be focused on fundamental theory, and students (particularly first generation) do not see the connection to their future career as engineers [15], [16], [20]. This effect is likely to be more pronounced for underrepresented and veteran students, who often are the first in their families to pursue a STEM degree. In addition, veteran students, similarly to non-traditional students, often have to balance time and financial commitments coming from already having a family, as well as the lack of a support network that 
will encourage them to forge ahead in their studies instead of finding a "safe" job (in fact, [21] found that many veterans often opt for traditional job sectors, e.g., security or law enforcement, in their haste to degree completion). What may appear to be an unusual struggle with fundamental courses (e.g., math, physics and chemistry), along with the missing linkage of STEM practice, results in students' losing motivation before they experience core curricula (typically at the junior/senior level) that are more directly connected to the subject of their choice. In contrast, by engaging in hands-on problem solving, undergraduate students experience a shift from passive to active learning [21]-[23], improve their design and laboratory skills [24][27], ultimately resulting in improved confidence and interest in STEM courses and careers [28]. More precisely, for first-year students, hands-on engineering courses retention rates have been shown to increase by an average of $16 \%$, across a number of similar programs at different institutions [26], [29]. Some programs report an even higher success rate in the retention of women and underrepresented minorities ([27], [29], [30]; for instance reference [29] found, respectively, a 27\%, 54\%, 36\% retention gain for women, Latino and African-American students). These gains are attributed to increased design, teamwork and communication skills [26], but also to the development of a peer support network.

For veterans, who already struggle to adjust to civilian life and manage a host of social and, sometimes, health issues [12], [13], [31], the gained confidence and sense of purpose from this kind of experience has the advantage of helping them better adapt to civilian life and longterm career goals. In addition, such hands-on learning experiences may be particularly beneficial since, according to a recent National Science Foundation-sponsored study, veteran students demonstrate little awareness about the professional value of their technical expertise, capacity, and training [21].

PIPELINES workforce experience hinges on projects that have been developed to challenge and motivate students by engaging them in authentic engineering design projects that have potential impact on real-world Navy problems. Indeed, the involvement of EXWC adds a crucial dimension to the program: projects submitted by Navy scientists and engineers pass the "relevance" test in the eyes of the students, who often lament the abstract nature of even lab courses offered in school [15]. In addition, students are given ownership of the project in that they are not only expected to implement tasks, but to propose solutions and come up with next steps. This requires careful crafting of the projects, which is done collaboratively by EXWC engineers and CSEP professionals (all with technical background), so that, although challenging, the projects have realistic expectations both in terms of student performance and progress over an eight-week time frame.

Overcoming the challenges faced during the internship and finishing their experience with tangible accomplishments was a big confidence boost for some students. Such sentiments were captured during evaluation interviews and exit surveys. In exit surveys, students expressed their confidence gained from working through an unfamiliar problem and realizing that they were capable of contributing to a solution. Other students described how valuable it was to have the opportunity to come up with their own ideas and test them, rather than just follow instructions. Others talked about how such real-world experiences would complement their coursework and help them gain a better understanding of their own interests and talents as well as the world outside of academia.

"Doing a project that's not necessarily in line with my major or whatever I'm doing in school kind of... I think it kind of gave me a little more confidence..."

"I believe my problem-solving skills have improved since beginning PIPELINES." 
The summer program also seemed to offer students a positive glimpse into the lives of Navy professionals through interactions with their Navy mentor, and the fact that work is mainly done at the Base. We designed such exposure to the Naval context believing it would be particularly beneficial to veteran and other underrepresented students who often are firstgeneration college-goers and, as such, may not have access to the role models who can offer first-hand academic and career guidance. In exit surveys, students confirmed that they found the exposure to a professional environment to be invaluable preparation for their futures, with $73 \%$ of participants reporting that they felt more interested in a civilian career in the Navy than they did at the start of the PIPELINES program.

"This program was my first internship and I was able to leave with positivity about my choice of career!"

\section{Leveraging Teamwork for Early Engagement in Engineering Design}

PIPELINES relies on teamwork to engage students in problems that otherwise would likely be too challenging for a single student, given the participants' very junior academic standing and the fact that for most of them PIPELINES represents the first internship experience. The team structure allows students to benefit from teammate skills and/or different background; gain confidence by brainstorming with peers in a non-threatening environment; and ultimately show more independence and ownership over the project.

Again, teams are composed of two CC students and one from a 4-year university. The 4year university student is hired directly by EXWC, goes through established selection procedures, and is assigned to their project based on major. CC students are hired through the PIPELINES ONR grant, and therefore recruited and selected by CSEP. The CC applicants had no minimum GPA requirement, and interns who satisfied the prerequisite 12 units of science/engineering classes were selected based on both academic accomplishments and other project-relevant skills acquired through jobs, volunteering, or other non-academic experience. Enthusiasm, maturity and communication skills were included in selection criteria and assessed through an interview (in-person or skype). The final selection of participating interns was made by the Navy mentors, who carefully reviewed the application materials and interview notes with guidance from the CSEP review committee. Of the $10 \mathrm{CC}$ interns, 6 were veterans, 2 female, 5 under-represented, 7 first-generation, 7 low-income, and 1 had disabilities.

The evaluation results revealed a consensus among the Navy mentors that the team structure was preferable to hosting single interns; all confirmed that the structure made interns more self-sufficient and productive.

In conversations with the program evaluator, students also acknowledged the value of teamwork: even with instances of compromise, they were still satisfied with what they were able to accomplish as a team. Students expressed that their experiences were stronger due to the presence of teammates to discuss and refine (and perhaps argue) about ideas with, and sometimes they could get more accomplished by delegating tasks and splitting up. One intern expressed the following:

"It was nice having a team, though. You work so much by yourself. Sometimes you need that experience, working on a team."

Most students agreed that working on a team, as opposed to working individually, helped them develop communication skills, learn to take or delegate responsibility, leverage the different strengths of teammates, and be mindful of different personalities. When asked if their 
professional development would have benefited more from an independent project, there was unanimous agreement that this was not the case.

Participants from 4-year universities admitted that they had initially felt skeptical about being paired with CC students. However, they had soon discovered that most of the CC students were older, and thus more mature and professional, and often military veterans demonstrated great practical perspectives. Regarding their experience working with veteran teammates, one intern remarked:

"I thought it was great... they were both very talented."

The experiences in working within team contexts was also explicitly recognized as valuable preparation to real-world work environments, by both mentors and participants. As one intern stated to the program evaluator:

"Working with my teammates prepared me to function on and contribute to a multidisciplinary team."

Although participants had in general positive feelings about the teamwork experience, some students acknowledged there being interpersonal difficulties that had to be solved before the team could be fully functional. Tensions arose when older team members, especially veterans, felt that their experience and professional skills were not appreciated or capitalized by their younger teammates. Also, the team structure, where the 4-year undergraduates were assigned the role of team leader by EXWC mentors, created friction in some cases, when/if older students felt that the teammate was not qualified to be the leader. Here is in the words of a participant:

"I think something they didn't take into account is that a lot of us are veterans. A lot of us are a lot older, and have a lot of work experience and life experience."

Establishing a formal training session on team communication and conflict management, as well as team building exercises, for both interns and mentors, will be a priority for the second year iteration of the program.

\section{Conclusions}

PIPELINES was designed to offer workforce-learning experiences that support undergraduate (both CC and 4-year students) students' development of career-critical skills while promoting engagement with real-world Naval design projects. Program design and implementation required a multi-tiered, interdisciplinary effort, in which each actor (educators, researchers, and Navy scientists and engineers) brings complementary knowledge and skills that, in concert, fostered the development of a successful postsecondary STEM program that provides real-world relevance to the students' experience. This interdisciplinary partnership is an example of how different entities can work together towards achieving their respective goals, all of which are focused on increasing and diversifying the engineering workforce in the U.S.

In this work, we have discussed findings from evaluation of our first iteration of the PIPELINES program and highlighted strategies we have found effective in developing and implementing an innovative program.

Acknowledgements: This material is based upon research supported by, or in part by, the U. S. Office of Naval Research under award number N000141512438. 


\section{References}

[1] US Congress Joint Economic Committee, "STEM Education: Preparing for the Jobs of the Future," 2012.

[2] R. B. Freeman, "Does Globalization of the Scientific/Engineering Workforce Threaten U.S. Economic Leadership?," in Innovation Policy and the Economy, 2005, no. Vol. 6.

[3] A. Carnevale, N. Smith, and M. Melton, "STEM: Science Technology Engineering Mathematics.," 2011.

[4] The Organisation for Economic Co-Operation and Development, "THE OECD JOBS STUDY Facts , Analysis , Strategies (1994).," 1994.

[5] “The U. S. STEM Undergraduate Model,” Business-Higher Educ. Forum, 2013.

[6] V. Bertram, "Better STEM Education with Project Lead the Way," Manufacturing Net News, 2012.

[7] J. P. Holdren and E. Lander, "Engage To Excel: Producing One Million Additional College Graduates With Degrees In Science, Technology, Engineering, And Mathematics," 2012.

[8] National Research Council, "Rising Above the Gathering Storm: Energizing and Employing America for a Brighter Economic Future," 2007.

[9] “Annual Benefits Reports, 2000 to 2012,” 2013.

[10] W. A. Lang and J. T. Powers, "Completing the Mission: A Pilot Study of Veteran Student Progress Toward Degree Attainment in the Post 9/11 Era," 2011.

[11] J. Scott, S. Himelstein, A. Perez, M. Baca, B. D. Lyman, B. D. Haley, and P. Macdougall, "Connecting Military Service and Civilian Life," Prep. by Chancellor's Off. Student Serv. Div. Off. Commun., 2011.

[12] M. Elliott, C. Gonzalez, and B. Larsen, "U.S. Military Veterans Transition to College: Combat, PTSD, and Alienation on Campus," J. Stud. Aff. Res. Pract., vol. 48, no. 3, pp. 279-296, 2011.

[13] R. Ackerman, D. DiRamio, and R. Mitchell, "Transitions: Combat Veterans As College Students," New Dir. Student Serv., vol. 126, 2009.

[14] "Veteran Students Task Force Final Report," 2010.

[15] S. Cui, Y. Wang, Y. Yang, F. M. Nave, and K. T. Harris, "Connecting Incoming Freshmen with Engineering through Hands-On Projects," Am. J. Eng. Educ., vol. 2, no. 2, pp. 31-42, 2011.

[16] “Transitioning Veterans to Engineering Related Careers," 2013.

[17] BEST, "A Bridge for All: Higher Education Design Principles to Broaden Participation in Science, Technology, Engineering and Mathematics," 2004.

[18] S. Olson and J. B. Labov, Community Colleges in the Evolving Stem Education 
Community - Summary of a Summit. National Academies Press, 2012.

[19] J. L. Green, A. Skukauskaite, and W. D. Baker, "Ethnography As Epistemology," in Research methods and methodologies in education, London, Sage, 2012, pp. 309-321.

[20] J. L. Steele, "Colleges Can Learn from For-Profits' Emphasis on the Consumer," 2012.

[21] L. Steinberg, C. Zoli, and N. Armstrong, "From Battlefield to Classroom: Findings, Barriers, and Pathways to Engineering for U.S. Servicemembers," 2011.

[22] M. Prince, "Does Active Learning Work? A Review of the Research," J. Eng. Educ., vol. 93, no. July, pp. 223-231, 2004.

[23] J. R. Goldberg, "Active learning in capstone design courses," IEEE Pulse, vol. 3, no. 3, pp. 54-56, 2012.

[24] S. G. Boodman, "Veterans find that their transition from combat to college can be difficult," Washington Post, 2011.

[25] K. W. Olson, "The GI Bill and higher education: Success and surprise," Am. Q., vol. 25, no. 5, pp. 596-610, 1973.

[26] J. Richardson and J. Dantzler, "Effect of a freshman engineering program on retention and academic performance," in 32nd Annual Frontiers in Education, 2002, vol. 3, pp. 16-22.

[27] V. Willson, T. Monogue, and C. Malave, "First Year Comparative Evaluation of the Texas A\&M Freshman Integrated Engineering Program," in Frontiers in Education Conference Proceedings, 1995, p. EAc13-19.

[28] M. T. Napoli and U. C. S. Barbara, "Engaging Community College Students in University Research," in 120th Annual ASEE Conference, 2013.

[29] D. W. Knight, L. E. Carlson, and J. F. Sullivan, "Staying in Engineering: First-Year Projects Course on Student Retention," in American Society for Engineering Education Annual Conference \& Exposition, 2003.

[30] M. Hoit and M. Ohland, "The Impact of a Discipline-Based Introduction to Engineering Course on Improving Retention,” J. Eng. Educ., vol. 87, no. 1, pp. 79-85, 1998.

[31] R. Glaser, J. T. Powers, and W. H. Zywiak, "Military Veterans at Universities: a Case of Culture Clash," Anthropol. News, vol. 50, no. 33, 2009. 